\title{
Poisson Ridge Regression Estimators: A Performance Test
}

\author{
Etaga Harrison Oghenekevwe ${ }^{1}$, Aforka Kenechukwu Florence ${ }^{1}$, Awopeju Kabiru Abidemi ${ }^{1}$, \\ Etaga Njideka Cecilia ${ }^{2}$
}

${ }^{1}$ Department of Statistics, Faculty of Physical Sciences, Nnamdi Azikiwe University, Awka, Nigeria

${ }^{2}$ Department of Educational Foundations, Faculty of Education, Nnamdi Azikiwe University, Awka, Nigeria

\section{Email address:}

ho.etaga@unizik.edu.ng (E. H. Oghenekevwe)

\section{To cite this article:}

Etaga Harrison Oghenekevwe, Aforka Kenechukwu Florence, Awopeju Kabiru Abidemi, Etaga Njideka Cecilia. Poisson Ridge Regression Estimators: A Performance Test. American Journal of Theoretical and Applied Statistics. Vol. 10, No. 2, 2021, pp. 111-121.

doi: $10.11648 /$ j.ajtas.20211002.13

Received: February 27, 2021; Accepted: March 15, 2021; Published: March 30, 2021

\begin{abstract}
In Multiple regression analysis, it is assumed that the independent variables are uncorrelated with one another, when such happen, the problem of multicollinearity occurs. Multicollinearity can create inaccurate estimates of the regression coefficients, inflate the standard errors of the regression coefficients, deflate the partial t-tests for the regression coefficients, give false $p$-values and degrade the predictability of the model. There are several methods to get rid of this problem and one of the most famous one is the ridge regression. The purpose of this research is to study the performance of some popular ridge regression estimators based on the effects of sample sizes and correlation levels on their Average Mean Square Error (AMSE) for Poisson Regression models in the presence of multicollinearity. As performance criteria, average MSE of k was used. A Monte Carlo simulation study was conducted to compare performance of Fifty (50) k estimators under four experimental conditions namely: correlation, Number of explanatory variables, sample size and intercept. From the results of the analysis as summarized in the Tables, the MSE of the estimators performed better in a lower explanatory variables $p$ and an increased intercept value. It was also observed that some estimators performed better on the average at all correlation levels, sample sizes, intercept values and explanatory variables than others.
\end{abstract}

Keywords: Multicollinearity, Ridge, Poisson, Estimators, Maximum Likelihood, Monte-Carlo Simulations, MSE

\section{Introduction}

In multiple regression analysis, predictions are made on one variable on the basis of several other variables. For example, suppose we were interested in predicting the level of exposure of an individual, variables such as age, gender, environment, academic qualifications and occupation might all contribute towards level of exposure. Another example could be when analyzing why people go to the cities; job opportunities, availability of basic amenities, schools and standard of living are important factors. In both examples, some or all covariates would be highly correlated. In the case of highly correlated explanatory variables, it is usually impossible to interpret the estimates of the individual coefficients. Such a problem is often referred to as the multicollinearity.

Multicollinearity exists whenever two or more of the predictor variables in a regression model are moderately or highly correlated. Multicollinearity can create inaccurate estimates of the regression coefficients, inflate the standard errors of the regression coefficients, deflate the partial t-tests for the regression coefficients, give false p-values and degrade the predictability of the model.

The Ridge Regression method is a well-known efficient remedial measure in the presence of multicollinearity. This method was first introduced by Schaeffer et al [16] and they have shown by both analytically and means of Monte Carlo simulations that this method has a smaller total Mean Square Error (MSE) than the OLS estimator. They suggested that a small positive number $(k>0)$ be added to the diagonal elements of the $X^{\prime} X$ matrix from the multiple regression, and the resulting estimator is obtained as

$$
\hat{\beta}_{R R}=\left(X^{\prime} X+k I_{p}\right)^{-1} X^{\prime} Y
$$

and this is known as the ridge regression (RR) estimator. Schaeffer et al [16] 
Different techniques for estimating the ridge parameter $\mathrm{k}$ have been suggested by a lots of researchers; [Hoerl and Kennard [6], Hoerl and Kennard [7], Algamal and Alanaz [3], Asar and Gen [4], Asar and Gen [5], Kaciranlar and Dawound [8], Kibra et al [12], Mansson and Shukur [13], Alkhamisi et al [1], Alkhamisi and Shukur [2], Muniz and Kibria [14], to mention but a few. However, the work on the estimation of the ridge parameter under the Poisson Regression model is limited. Schaeffer et al [16] worked on the Simple Poisson Regression model, Muniz and Kibria [14] considered the Poisson RR models, Kibria et al [11] generalized some estimators of the ridge parameters proposed for logistic regression by Kibria et al [12] for Poisson ridge regression and Zaldival [17] considered the performance of some Poisson Ridge Regression Estimators.

One of the standard statistical method for analyzing count data is the Poisson Regression (PR) model. This model has found a widespread use in microeconometrics when the dependent variable $\mathrm{y}$ of the regression model is Poisson distributed. In the presence of multicollinearity, when estimating the parameters for Poisson regression model using the maximum likelihood (ML) method, the estimated parameters become instable with high variance, resulting in an increase in the probability of conducting a Type II error in any hypothesis testing regarding the estimated parameters Kibria et al [11].

Several techniques for estimating the ridge parameter $\mathrm{k}$ have been suggested by a lot of researchers;

The purpose of this article is to determine the best estimators among 50 selected ridge parameters estimators (k), based on the effect of sample sizes and levels of correlation on the performance of the 50 selected estimators. The performance of the estimators is judged by the Average MSE of $k$.

\section{Literature}

Zaldivar [17] investigated some Ridge Regression (RR) estimators for estimating the ridge parameter $k$ for the poisson regression model and proposed five new estimators. A simulation study was conducted to compare the performance of some of the estimators in literature with the newly proposed estimators using the average Mean Square Error (MSE), percentage of times Poisson Ridge Regression (PRR) outperforms MLE and the Average Mean Absolute Percentage Error (AMAPE) as performance criteria, these five new estimators performed well, producing small MSE values. A real life data was also used to illustrate the findings.

Kibria et al [11] conducted a simulation study of some biasing parameters for the ridge type estimation of Poisson regression, they generalized some estimators proposed for logistic regression by Kibria [12]. In the work they included the average value of $k$ and the standard deviation of $k$ as performance criteria aside the Mean Square Error (MSE), these performance criteria are very informative because if several estimators have equal estimated MSE, then those with low average value and low standard deviation of $k$ should be preferred.

Muniz and Kibria [14] proposed a Poisson Ridge Regression (PRR) estimator, by means of Monte Carlo simulations they evaluated the traditional ML estimator and this new method using different estimators of the ridge parameter $k$. The result from the simulation study showed that the sample size, the value of the intercept, the number of independent variables and the correlation between the independent variables are important factors for the performance of the different estimation methods. The result also showed that the proposed PRR method, regardless which ridge estimator used, has a lower MSE than the ML method for all different situations that has been evaluated. Many researchers, Hoarl and Kennard [6], have worked on Regression and Poison estimator and their conclusion varied. Hoerl and Kennard [6], Hoerl and Kennard [7], Algamal and Alanaz [3], Asar and Gen [4], Asar and Gen [5], Kaciranlar and Dawound [8], Kibra et al [12], Mansson and Shukur [13], Alkhamisi et al [1], Alkhamisi and Shukur [2], Khalaf Ghazi [9], Kibria [10].

\section{Methodology}

This section starts by defining the Poisson Regression model and the traditional ML estimation method.

\subsection{The Poisson Regression}

The Poisson Regression is similar to the regular multiple regression except that the dependent variable $(Y)$ is an observed count that follows the poisson distribution. Thus the possible values of $(Y)$ are the non-negative integers: $0,1,2$, 3 , and so on. The model of this regression is given as:

$$
y_{i}=u_{i}+\varepsilon_{i}(i=1,2, \ldots, n)
$$

Where $y_{i}$ is an $n \times 1$ vector of responses that is poisson distributed, $u_{i}=\exp \left(x_{i} \beta\right)$ is an $n \times(p+1)$ data matrix with $p$ explanatory variables, and $\varepsilon_{i}$ is an $n \times 1$ vector of random errors.

The parameters of this model are estimated using the Maximum Likelihood (ML) method and the following iterated weighted least-square algorithm:

$$
\hat{\beta}_{M L}=\left(X^{\prime} \widehat{W} X\right)^{-1} X^{\prime} \widehat{W} \hat{z},
$$

Where $\hat{z}$ is a vector where the $i$ th element equals $\hat{z}_{i}=$ $\log \left(\hat{\mu}_{i}\right)+\frac{y_{i-\widehat{\mu}_{i}}}{\widehat{\mu}_{i}}$ and $\widehat{W}$ is a matrix where the off- diagonal elements are equal to zero and the $i$ th diagonal element is equal to $\hat{\mu}_{i}$. Where $\hat{\mu}_{i}=\exp \left(x_{i} \beta\right)$ and $x_{i}$ is the $i^{\text {th }}$ row of $X$.

\subsection{Poisson Ridge Regression}

In the presence of multicollinearity, the weighted matrix of cross products $\left(X^{\prime} \widehat{W} X\right)$ is near singular. For this model, the following extension of the linear ridge regression estimator (RRE) was proposed by Mansson and Shukur [13].

$$
\hat{\beta}_{R R}=\left(X^{\prime} \widehat{W} X+k I_{P}\right)^{-1}\left(X^{\prime} \widehat{W} X \hat{\beta}_{M L}\right) .
$$


They showed that the above estimator approximately reduces the increase of the weighted Sum of Squared Error. The reason for Poisson Ridge Regression (PRR) is to find a value of $\mathrm{k}$ that is large enough, but not so big that it causes a lot of bias. When such a $\mathrm{k}$ is found, then the MSE of the RIDGE regression (RR) estimator will be smaller than that of the ML estimator. The MSE of RR equals

$$
\begin{gathered}
E\left(L_{R R}^{2}\right)=E\left(\beta_{R R}-\beta\right)^{\prime\left(\beta_{R R}-\beta\right)}=\sum_{j=1}^{J} \frac{\lambda_{j}}{\left(\lambda_{j}+k\right)^{2}}+ \\
k^{2} \sum_{j=1}^{J} \frac{\alpha_{j}}{\left(\lambda_{j}+k\right)^{2}}
\end{gathered}
$$

While the MSE of the ML estimator is

$$
E\left(L_{M L}^{2}\right)=E\left(\beta_{M L}-\beta\right)^{\prime}\left(\beta_{M L}-\beta\right)=\operatorname{tr}\left(X^{\prime} \widehat{W} X\right)^{-1}=\sum_{j=1}^{J} \frac{1}{\lambda_{j}}(6)
$$

\subsection{Some Methods for Estimating the Ridge Parameter (k)}

Various methods have been suggested by different researchers for estimating the ridge parameter $k$.

Hoerl and Kennard [6] were the first to propose a ridge parameter estimator and their estimator formed the basis upon which other estimators were proposed. The first estimator is

$$
k_{1}=\hat{k}_{H K 1}=\frac{\hat{\sigma}^{2}}{\hat{\alpha}_{\max }^{2}}
$$

Where $\hat{\sigma}^{2}=\frac{\sum_{i=1}^{n}\left(y_{i}-\widehat{\mu}_{i}\right)^{2}}{n-p-1}$, and $\hat{\alpha}_{\max }$ is the maximum element of $\hat{\alpha}$, where $\hat{\alpha}=U^{\prime} \hat{\beta}_{M L}$ and $U$ is the matrix whose columns are the eigenvectors of $X^{\prime} \widehat{W} X$.

Schaeffer et al [16] proposed the following for logistic ridge regression

$$
k_{2}=\hat{k}_{H K M}=\frac{1}{\widehat{\alpha}_{\max }^{2}}
$$

Kibria (2003):

$$
\begin{aligned}
& k_{3}=\hat{k}_{G M}=\frac{\hat{\sigma}^{2}}{\left(\Pi_{j=1}^{p} \widehat{\alpha}_{j}^{2}\right)^{\frac{1}{P}}} \\
& k_{4}=\hat{k}_{M E D}=\operatorname{Median}\left\{m_{j}^{2}\right\},
\end{aligned}
$$

Where $m_{j}=\sqrt{\frac{\widehat{\sigma}^{2}}{\widehat{\alpha}_{j}^{2}}}$.

Muniz et al [14] and Kibria et al [12] proposed $\mathrm{k}_{5}$ to $\mathrm{k}_{8}$ given as

$$
\begin{gathered}
k_{5} \max =\left(\frac{1}{m_{j}}\right) \\
k_{6} \max =\left(m_{j}\right) \\
k_{7}=\prod_{j=1}^{p}\left(\frac{1}{m_{j}}\right)^{\frac{1}{p}} \\
k_{8}=\text { median }\left(\frac{1}{m_{j}}\right)
\end{gathered}
$$

Kibria et al [11] proposed $\mathrm{k}_{9}$ to $\mathrm{k}_{16}$ given as

$$
\begin{gathered}
k_{9}=\prod_{j=1}^{p}\left(m_{j}\right)^{\frac{1}{p}} \\
k_{10}=\operatorname{median}\left(m_{j}\right) \\
k_{11}=\max \left(\frac{1}{q_{j}}\right) \\
k_{12}=\max \left(q_{j}\right) \\
k_{13}=\prod_{j=1}^{p}\left(\frac{1}{q_{j}}\right)^{\frac{1}{p}} \\
k_{14}=\prod_{j=1}^{p}\left(q_{j}\right)^{\frac{1}{p}} \\
k_{15}=\text { median }\left(\frac{1}{q_{j}}\right) \\
k_{16}=\operatorname{median}\left(q_{j}\right)
\end{gathered}
$$

Where $q_{j}=\frac{\lambda_{\max }}{(n-p) \widehat{\sigma}^{2}+\lambda_{\max } \widehat{\alpha}_{j}^{2}}$, where $\lambda_{\max }$ is the maximum eigenvalue of $X^{\prime} \widehat{W} X$.

Zaldival [17] used the following in her work, 'on the performance of some Poisson Ridge estimators'

$$
\begin{gathered}
k_{17}=K_{Y 1}=\frac{1}{p} \sum_{j=1}^{p} \sqrt{\frac{1}{\lambda_{j} \hat{\alpha}_{j}^{2}}} \\
k_{18}=K_{Y 2}=\left(\prod_{j=1}^{p} \sqrt{\frac{1}{\lambda_{j} \widehat{\alpha}_{j}^{2}}}\right)^{\frac{1}{p}} \\
k_{19}=K_{Y 3}=\operatorname{median}\left(\sqrt{\frac{1}{\lambda_{j} \widehat{\alpha}_{j}^{2}}}\right), j=1,2, \ldots, p \\
k_{20}=K_{Y 4}=\max \left(\sqrt{\frac{1}{\lambda_{j} \widehat{\alpha}_{j}^{2}}}\right), j=1,2, \ldots, p \\
k_{21}=K_{Y 5}=\operatorname{median}\left(\sqrt{\lambda_{j} \hat{\alpha}_{j}^{2}}\right), j=1,2, \ldots, p \\
k_{22}=K_{Y 6}=\max \left(\sqrt{\lambda_{j} \hat{\alpha}_{j}^{2}}\right), j=1,2, \ldots, p \\
k_{23}=K_{Y 7}=\frac{1}{p} \sum_{j=1}^{p}\left(\sqrt{\lambda_{j} \hat{\alpha}_{j}^{2}}\right) \\
k_{24}=K_{Y 8}=\frac{p}{\sum_{j=1}^{p} \sqrt{\left(\sqrt{\lambda_{j} \widehat{\alpha}_{j}^{2}}\right)}} \\
K_{Y 9}=\frac{p}{\sum_{j=1}^{p} \sqrt{\frac{1}{\lambda_{j} \widehat{\alpha}_{j}^{2}}}}
\end{gathered}
$$

where $\lambda_{j}$ is the $j^{\text {th }}$ eigenvalue of $X^{\prime} \widehat{W} X$.

Muniz et al [14] proposed the following estimators, based on square root transformations:

$$
k_{26}=\operatorname{Max}\left\{\sqrt{\hat{\alpha}_{j}^{2}}\right\}
$$




$$
\begin{aligned}
& k_{27}=\operatorname{Median}\left\{\sqrt{\hat{\alpha}_{j}^{2}}\right\} \\
& k_{28}=\operatorname{Max}\left\{\frac{1}{\sqrt{\widehat{\alpha}_{j}^{2}}}\right\} \\
& k_{29}=\left(\prod_{j=1}^{p} \sqrt{\hat{\alpha}_{j}^{2}}\right)^{1 / p} \\
& k_{30}=\left(\prod_{j=1}^{p} \sqrt{\frac{1}{\widehat{\alpha}_{j}^{2}}}\right)^{1 / p} \\
& k_{31}=\operatorname{Median}\left\{\frac{1}{\sqrt{\widehat{\alpha}_{j}^{2}}}\right\} \\
& k_{32}=\frac{p}{\sum_{j=1}^{p} \lambda_{j} \widehat{\alpha}_{j}^{2}} \\
& k_{33}=\frac{\lambda_{\max }}{(n-p)+\lambda_{\max } \widehat{\alpha}_{\max }^{2}} \\
& k_{34}=\frac{1}{p} \sum_{j=1}^{p}\left(\frac{\lambda_{j}}{(n-p)+\lambda_{j} \widehat{\alpha}_{j}^{2}}\right) \\
& k_{35}=\operatorname{Max}\left\{\frac{\lambda_{j}}{(n-p)+\lambda_{j} \widehat{\alpha}_{j}^{2}}\right\} \\
& k_{36}=\operatorname{Median}\left\{\frac{\lambda_{j}}{(n-p)+\lambda_{j} \widehat{\alpha}_{j}^{2}}\right\} \\
& k_{37}=\prod_{j=1}^{p}\left\{\frac{\lambda_{j}}{(n-p)+\lambda_{j} \widehat{\alpha}_{j}^{2}}\right\}^{1 / p} \\
& k_{38}=\frac{1}{\widehat{\alpha}_{\max }^{2}}+\frac{1}{\lambda_{\max }} \\
& k_{39}=\operatorname{Max}\left\{\frac{1}{\widehat{\alpha}_{j}^{2}}+\frac{1}{\lambda_{j}}\right\} \\
& k_{40}=\frac{1}{\operatorname{Min}\left\{\frac{1}{\hat{\alpha}_{j}^{2}}+\frac{1}{\lambda_{j}}\right\}} \\
& k_{41}=\operatorname{Max}\left\{\sqrt{\frac{(n-p)+\lambda_{\max } \widehat{\alpha}_{j}^{2}}{\lambda_{\max }}}\right\} \\
& k_{42}=\prod_{j=1}^{p}\left\{\sqrt{\frac{(n-p)+\lambda_{\max } \widehat{\alpha}_{j}^{2}}{\lambda_{\max }}}\right\}^{1 / p} \\
& k_{43}=\operatorname{Median}\left\{\sqrt{\frac{(n-p)+\lambda_{\max } \widehat{\alpha}_{j}^{2}}{\lambda_{\max }}}\right\} \\
& k_{44}=\prod_{j=1}^{p}\left(\frac{(n-p)+\lambda_{\max } \widehat{\alpha}_{j}^{2}}{\lambda_{\max }}\right)^{1 / p} \\
& k_{45}=\operatorname{Max}\left\{\sqrt{\frac{\lambda_{\max }}{(n-p)+\lambda_{\max } \widehat{\alpha}_{j}^{2}}}\right\}
\end{aligned}
$$

\section{Results / Findings}

In this work, Multicollinearity was discussed. Some

\subsection{Performance of the Estimators}

To investigate the performance of the Poisson Ridge RR and the Maximum Likelihood ML method, the Average MSE (AMSE) are obtained using the following equation:

$$
\mathrm{AMSE}=\frac{\sum_{i=1}^{r} S E_{i}}{r}=\frac{\sum_{i=1}^{r}(\widehat{\beta}-\beta)_{i}^{\prime}(\widehat{\beta}-\beta)_{i}}{r}
$$

where $r=$ number of replicates $=1000$

Where $\hat{\beta}$ is the estimator of $\beta$ obtained from ML or PRR and $\mathrm{SE}$ is the squared error.

\subsection{The Monte Carlo Simulation}

This section consists of a brief description of how the data is generated and the factors varied in the simulation study.

\subsection{The Design of the Experiment}

The dependent variable (y) of the Poisson Regression Model is generated in $\mathrm{R}$ using pseudo-random numbers from the poisson distribution, with

$$
\text { mean }\left(\mu_{i}\right)=e^{\beta_{0}+\beta_{1} x_{i 1}+\cdots+\beta_{p} x_{i p}} ; i=1,2, \ldots, \mathrm{n}
$$

The parameter values in equ (4.1) are chosen so that $\sum_{j=1}^{p} \beta_{j}^{2}=1$ and $\beta_{1}=\beta_{2}=\cdots=\beta_{p}$. These are common restrictions in many simulation studies (zaldivar, 2018).

The degrees of freedom are defined as $d f=n-p-$ 1. the value of $\beta_{o}=-1,0,1$.

The independent variables are generated as follows:

$$
x_{i j}=\sqrt{1-\rho^{2}} z_{i j}+\rho z_{i p} ; i=1,2, \ldots, n ; j=1,2, \ldots, p
$$

Where $z_{i j}$ are pseudo-random numbers from the standard normal distribution, $\rho^{2}$ represents the degree of correlation. In the design of the experiment, three values of $\rho^{2}$ are considered which are $0.85,0.90,0.99$. Other factors that were varied includes sample size $(n)=50$ and 200 , number of explanatory variables $(p)=4$ and 8 and intercept value $\left(\beta_{0}\right)$ $=-1,0,1$. 
selected Ridge Regression (RR) estimators for estimating the ridge regression parameter $\mathrm{k}$ for Poisson Regression model were investigated for correlated variables. Monte-Carlo Simulation was carried out at two explanatory variables, $p=4$ and $8, \quad$ three correlation levels $\rho=0.85,0.90$, and 0.99 and intercept, $\beta_{o}=-1,0$ and 1 with 50 selected estimators. For each combination, 1000 replications were performed, the performance of this estimators have been evaluated using the Average MSE.

From the simulated results, it was shown that some estimators are affected by sample sizes while some are not affected by sample sizes. For examples, at $\rho=0.85, p=4$ and $\beta_{o}=-1$, the MSE of the estimators are drastically affected by the sample size, while at $\rho=0.90$, and 0.99 for $p=4$ and $\beta_{o}=-1$, the estimators are not affected by the sample sizes. For $\rho=0.85$ and $0.99, p=4$ and $\beta_{o}=0$, the MSE of the estimators are affected by the sample sizes, while it not affected by the sample sizes at $\rho=0.90$. For $p=4$, $\beta_{o}=1$ and for all correlation levels, except for $\rho=$ 0.99 , the MSE of the estimators are not affected by the sample sizes.

For $p=8, \beta_{o}=-1$ and for all correlation levels, the MSE of the estimators are not affected by the sample sizes. For $=8, \beta_{o}=0$, and $\rho=0.85$ and 0.90 , the MSE of the estimators are not affected by the sample sizes except for $\rho=0.99$. For $p=8, \beta_{o}=1$, the MSE of the estimators are not affected by the sample sizes at all levels of correlation.

Table 1. Ranked Mean Square Error of estimators for $p=4$ and $\beta=-1$ at varying correlation levels and sample sizes.

\begin{tabular}{|c|c|c|c|c|c|c|c|c|c|c|c|c|c|}
\hline \multirow[b]{2}{*}{ Estimators } & \multicolumn{4}{|l|}{$\rho=0.85$} & \multicolumn{4}{|l|}{$\rho=0.90$} & \multicolumn{5}{|l|}{$\rho=0.99$} \\
\hline & $\begin{array}{l}\text { Sample } \\
50(0.85)\end{array}$ & R1 & $\begin{array}{l}\text { Sample } \\
200(0.85)\end{array}$ & $\mathbf{R 2}$ & $\begin{array}{l}\text { Sample } \\
50(0.90)\end{array}$ & $\mathbf{R 3}$ & $\begin{array}{l}\text { Sample } \\
200(0.90)\end{array}$ & R4 & $\begin{array}{l}\text { Sample } \\
50(0.99)\end{array}$ & R5 & $\begin{array}{l}\text { Sample } \\
200(0.99)\end{array}$ & R6 & $\begin{array}{l}\text { Total } \\
\text { MSE }\end{array}$ \\
\hline $\mathrm{k} 14$ & 0.0002 & 3 & 0.6 & 32 & 0.0003 & 3 & 0 & 3 & 0 & 1 & 0 & 1 & 0.6005 \\
\hline k12 & 0.0002 & 2 & 0.003 & 13 & 0.0004 & 4 & 0 & 4 & 0 & 2 & 0 & 2 & 0.0036 \\
\hline k16 & 0.0002 & 4 & 12.5 & 43 & 0.0004 & 5 & 0 & 5 & 0 & 3 & 0 & 3 & 12.5006 \\
\hline k36 & 0.0001 & 1 & 0.35 & 31 & 0 & 1 & 0 & 1 & 0.0001 & 4 & 0 & 4 & 0.3502 \\
\hline k37 & 0.0004 & 5 & 2.37 & 38 & 0 & 2 & 0 & 2 & 0.0004 & 5 & 0 & 5 & 2.3708 \\
\hline k45 & 0.0181 & 17 & 0.005 & 16 & 0.0015 & 7 & 0 & 7 & 0.0172 & 16 & 0 & 7 & 0.0418 \\
\hline k46 & 0.0176 & 16 & 0.001 & 11 & 0.0069 & 12 & 0 & 11 & 0.0173 & 17 & 0 & 8 & 0.0428 \\
\hline k35 & 0.0265 & 21 & 0.29 & 30 & 0.0036 & 10 & 0 & 10 & 0.0254 & 22 & 0 & 9 & 0.3455 \\
\hline k47 & 0.0258 & 20 & 0 & 4 & 0.0114 & 16 & 0 & 12 & 0.0254 & 23 & 0 & 10 & 0.0626 \\
\hline k43 & 0.255 & 29 & 1.19 & 36 & 0.0015 & 8 & 0 & 8 & 0.242 & 26 & 0 & 11 & 1.6885 \\
\hline $\mathrm{k} 42$ & 0.255 & 27 & 0 & 6 & 0.0033 & 9 & 0 & 9 & 0.242 & 27 & 0 & 12 & 0.5003 \\
\hline k33 & 0.0257 & 19 & 1.85 & 37 & 0.0208 & 20 & 0.001 & 14 & 0.0253 & 21 & 0.001 & 13 & 1.9238 \\
\hline k41 & 0.255 & 28 & 0 & 7 & 0.0111 & 15 & 0.001 & 13 & 0.242 & 28 & 0.001 & 14 & 0.5101 \\
\hline k8 & 0.0053 & 7 & 21.1 & 41 & 0.0094 & 14 & 0.004 & 16 & 0.0043 & 7 & 0.004 & 16 & 21.127 \\
\hline $\mathrm{k} 40$ & 0.0043 & 6 & 0 & 1 & 0.0054 & 11 & 0.005 & 17 & 0.0035 & 6 & 0.005 & 17 & 0.0232 \\
\hline k21 & 0.0084 & 10 & 0 & 2 & 0.0141 & 17 & 0.006 & 18 & 0.006 & 9 & 0.006 & 18 & 0.0405 \\
\hline k27 & 0.01 & 11 & 0.03 & 26 & 0.0179 & 19 & 0.008 & 20 & 0.0081 & 11 & 0.007 & 19 & 0.081 \\
\hline k29 & 0.0101 & 12 & 0.227 & 29 & 0.0175 & 18 & 0.007 & 19 & 0.0084 & 12 & 0.007 & 20 & 0.277 \\
\hline k18 & 0.0121 & 13 & 12.2 & 42 & 0.0209 & 21 & 0.009 & 21 & 0.01 & 13 & 0.008 & 21 & 12.26 \\
\hline $\mathrm{k} 23$ & 0.0143 & 14 & 0.009 & 20 & 0.0228 & 22 & 0.01 & 22 & 0.012 & 15 & 0.01 & 22 & 0.0781 \\
\hline k5 & 0.0164 & 15 & 0.005 & 15 & 0.0279 & 23 & 0.012 & 23 & 0.0115 & 14 & 0.012 & 23 & 0.0848 \\
\hline k19 & 0.021 & 18 & 0 & 3 & 0.0368 & 24 & 0.019 & 24 & 0.0176 & 18 & 0.019 & 24 & 0.1134 \\
\hline k17 & 0.0311 & 23 & 0 & 5 & 0.055 & 26 & 0.024 & 25 & 0.0234 & 20 & 0.024 & 25 & 0.1575 \\
\hline k26 & 0.031 & 22 & 0.006 & 17 & 0.0531 & 25 & 0.026 & 26 & 0.0217 & 19 & 0.025 & 26 & 0.1628 \\
\hline $\mathrm{k} 22$ & 0.0403 & 24 & 0.024 & 24 & 0.0648 & 27 & 0.029 & 27 & 0.0349 & 24 & 0.029 & 27 & 0.222 \\
\hline $\mathrm{k} 20$ & 0.0903 & 25 & 12.5 & 44 & 0.158 & 30 & 0.07 & 28 & 0.067 & 25 & 0.068 & 28 & 12.9533 \\
\hline $\mathrm{k} 48$ & 0.4 & 31 & 0 & 8 & 0.128 & 29 & 0.104 & 29 & 0.94 & 33 & 0.113 & 29 & 1.685 \\
\hline $\mathrm{k} 25$ & 0.212 & 26 & 0.07 & 27 & 0.11 & 28 & 0.111 & 30 & 0.269 & 29 & 0.115 & 30 & 0.887 \\
\hline k30 & 0.438 & 32 & 0.106 & 28 & 0.264 & 33 & 0.293 & 33 & 0.565 & 31 & 0.287 & 33 & 1.953 \\
\hline k31 & 0.458 & 33 & 0.025 & 25 & 0.38 & 35 & 0.353 & 34 & 0.592 & 32 & 0.342 & 34 & 2.15 \\
\hline k49 & 0.568 & 35 & 14.0 & 45 & 0.32 & 34 & 0.405 & 35 & 1.37 & 38 & 0.388 & 35 & 17.051 \\
\hline k9 & 0.95 & 36 & 0.731 & 33 & 0.588 & 36 & 0.609 & 36 & 1.29 & 35 & 0.597 & 36 & 4.765 \\
\hline k10 & 1 & 37 & 0.012 & 22 & 0.872 & 39 & 0.738 & 37 & 1.35 & 36 & 0.717 & 37 & 4.689 \\
\hline k4 & 1 & 38 & 27.4 & 50 & 0.872 & 40 & 0.738 & 38 & 1.35 & 37 & 0.717 & 38 & 32.077 \\
\hline k2 & 4.11 & 42 & 23.5 & 49 & 1.94 & 43 & 1.29 & 40 & 3.79 & 40 & 1.38 & 39 & 36.01 \\
\hline k38 & 4.11 & 41 & 0.001 & 12 & 1.94 & 42 & 1.29 & 39 & 12 & 44 & 1.38 & 40 & 20.721 \\
\hline k28 & 3.2 & 40 & 0.01 & 21 & 0.7 & 37 & 1.82 & 41 & 3.62 & 39 & 1.74 & 41 & 11.09 \\
\hline k32 & 1.77 & 39 & 0.007 & 19 & 0.77 & 38 & 2.38 & 42 & 4.15 & 41 & 2.3 & 42 & 11.377 \\
\hline k6 & 6.24 & 44 & 4.99 & 40 & 1.37 & 41 & 3.99 & 43 & 7.09 & 43 & 3.81 & 43 & 27.49 \\
\hline k1 & 18.5 & 45 & 28.5 & 51 & 7.64 & 44 & 5.62 & 44 & 17.7 & 45 & 6.02 & 44 & 83.98 \\
\hline k3 & 49.6 & 47 & 19.9 & 48 & 17.6 & 46 & 21.6 & 45 & 44.5 & 47 & 21.1 & 45 & 174.3 \\
\hline ML & 89.0058 & 51 & 193.112 & 47 & 46.0752 & 51 & 88.049 & 47 & 76.0055 & 51 & 100.005 & 46 & 592.2525 \\
\hline k13 & 71.1 & 48 & 0.004 & 14 & 38.9 & 48 & 87 & 46 & 64.8 & 50 & 105 & 47 & 366.804 \\
\hline
\end{tabular}




\begin{tabular}{|c|c|c|c|c|c|c|c|c|c|c|c|c|c|}
\hline \multirow[b]{2}{*}{ Estimators } & \multicolumn{4}{|l|}{$\rho=0.85$} & \multicolumn{4}{|l|}{$\rho=0.90$} & \multicolumn{5}{|l|}{$\rho=0.99$} \\
\hline & $\begin{array}{l}\text { Sample } \\
\text { 50(0.85) }\end{array}$ & R1 & $\begin{array}{l}\text { Sample } \\
200(0.85)\end{array}$ & $\mathbf{R 2}$ & $\begin{array}{l}\text { Sample } \\
\text { 50(0.90) }\end{array}$ & $\mathbf{R 3}$ & $\begin{array}{l}\text { Sample } \\
200(0.90)\end{array}$ & R4 & $\begin{array}{l}\text { Sample } \\
50(0.99)\end{array}$ & R5 & $\begin{array}{l}\text { Sample } \\
200(0.99)\end{array}$ & R6 & $\begin{array}{l}\text { Total } \\
\text { MSE }\end{array}$ \\
\hline $\mathrm{k} 15$ & 71.1 & 49 & 0.731 & 34 & 38.9 & 49 & 89 & 48 & 64.7 & 48 & 107 & 48 & 371.431 \\
\hline k11 & 71.1 & 50 & 4.07 & 39 & 39.7 & 50 & 89.4 & 49 & 64.7 & 49 & 107 & 49 & 375.97 \\
\hline k44 & 5.83 & 43 & 16.9 & 46 & 37 & 47 & 96.8 & 50 & 5.63 & 42 & 117 & 50 & 279.16 \\
\hline k39 & 22.2 & 46 & 0 & 10 & 13 & 45 & 166 & 51 & 21.2 & 46 & 157 & 51 & 379.4 \\
\hline
\end{tabular}

Table 2. Ranked Mean Square Error of estimators for $p=4$ and $\beta=0$ at varying correlation levels and sample sizes.

\begin{tabular}{|c|c|c|c|c|c|c|c|c|c|c|c|c|c|}
\hline \multirow[b]{2}{*}{ Estimators } & \multicolumn{4}{|l|}{$\rho=0.85$} & \multicolumn{4}{|l|}{$\rho=0.90$} & \multicolumn{5}{|l|}{$\rho=0.99$} \\
\hline & $\begin{array}{l}\text { Sample } \\
50(0.85)\end{array}$ & R1 & $\begin{array}{l}\text { Sample } \\
200(0.85)\end{array}$ & $\mathbf{R 2}$ & $\begin{array}{l}\text { Sample } \\
50(0.90)\end{array}$ & $\mathbf{R 3}$ & $\begin{array}{l}\text { Sample } \\
200(0.90) \\
\end{array}$ & R4 & $\begin{array}{l}\text { Sample } \\
50(0.99)\end{array}$ & R5 & $\begin{array}{l}\text { Sample } \\
200(0.99)\end{array}$ & R6 & $\begin{array}{l}\text { Total } \\
\text { MSE }\end{array}$ \\
\hline k36 & 0.0178 & 17 & 0 & 3 & 0 & 2 & 0 & 2 & 0 & 1 & 0 & 1 & 0.0178 \\
\hline k37 & 0.0002 & 1 & 0 & 1 & 0 & 1 & 0 & 1 & 0.0001 & 2 & 0 & 2 & 0.0003 \\
\hline k14 & 7.24 & 45 & 0 & 5 & 0.0004 & 4 & 0 & 4 & 0.0006 & 4 & 0 & 3 & 7.241 \\
\hline k16 & 7.23 & 44 & 0 & 4 & 0.0004 & 3 & 0 & 3 & 0.0007 & 5 & 0 & 4 & 7.2311 \\
\hline k12 & 7.25 & 46 & 0 & 6 & 0.0004 & 5 & 0 & 5 & 0.0008 & 6 & 0 & 5 & 7.2512 \\
\hline k34 & 0.0107 & 10 & 0 & 2 & 0.0009 & 6 & 0 & 6 & 0.0039 & 9 & 0 & 6 & 0.0155 \\
\hline k45 & 0.0036 & 6 & 0.0001 & 7 & 0.0016 & 7 & 0 & 7 & 0.0083 & 13 & 0 & 7 & 0.0136 \\
\hline k46 & 0.0059 & 7 & 0.0005 & 12 & 0.0069 & 12 & 0 & 11 & 0.0066 & 11 & 0.001 & 8 & 0.0209 \\
\hline k47 & 0.0087 & 9 & 0.0004 & 11 & 0.0111 & 16 & 0 & 12 & 0.0067 & 12 & 0.001 & 9 & 0.0279 \\
\hline k42 & 0.0026 & 5 & 0.0003 & 10 & 0.0035 & 9 & 0 & 9 & 0.0139 & 14 & 0.001 & 10 & 0.0213 \\
\hline k43 & 0.0017 & 2 & 0.0002 & 9 & 0.0016 & 8 & 0 & 8 & 0.014 & 15 & 0.001 & 11 & 0.0185 \\
\hline k35 & 0.013 & 11 & 0.0001 & 8 & 0.0037 & 10 & 0 & 10 & 0.0157 & 17 & 0.001 & 12 & 0.0335 \\
\hline k33 & 0.252 & 33 & 0.0013 & 14 & 0.0206 & 21 & 0.001 & 14 & 0.0244 & 23 & 0.001 & 13 & 0.3003 \\
\hline k41 & 0.123 & 28 & 0.0012 & 13 & 0.0116 & 17 & 0.001 & 13 & 0.0514 & 31 & 0.004 & 14 & 0.1922 \\
\hline k7 & 3.27 & 41 & 0.0043 & 16 & 0.0093 & 13 & 0.003 & 15 & 0.021 & 19 & 0.006 & 15 & 3.3136 \\
\hline k8 & 0.0077 & 8 & 0.0039 & 15 & 0.0094 & 14 & 0.004 & 16 & 0.0237 & 22 & 0.006 & 16 & 0.0547 \\
\hline k21 & 0.0441 & 19 & 0.0064 & 20 & 0.0136 & 18 & 0.006 & 18 & 0.0303 & 24 & 0.013 & 17 & 0.1134 \\
\hline k29 & 1.65 & 40 & 0.0081 & 22 & 0.0177 & 19 & 0.007 & 19 & 0.041 & 25 & 0.013 & 18 & 1.7368 \\
\hline k27 & 0.0167 & 16 & 0.0073 & 21 & 0.018 & 20 & 0.007 & 20 & 0.0455 & 26 & 0.013 & 19 & 0.1075 \\
\hline k40 & 0.0024 & 4 & 0.102 & 33 & 0.0046 & 11 & 0.005 & 17 & 0.0634 & 33 & 0.013 & 20 & 0.1904 \\
\hline k18 & 0.0163 & 15 & 0.0096 & 23 & 0.0211 & 22 & 0.009 & 21 & 0.0489 & 30 & 0.015 & 21 & 0.1199 \\
\hline k5 & 0.412 & 34 & 0.0127 & 25 & 0.0272 & 24 & 0.011 & 23 & 0.0457 & 27 & 0.02 & 22 & 0.5286 \\
\hline k23 & 0.0148 & 14 & 0.0118 & 24 & 0.0225 & 23 & 0.01 & 22 & 0.0697 & 34 & 0.027 & 23 & 0.1558 \\
\hline k19 & 0.0487 & 20 & 0.0152 & 26 & 0.0368 & 25 & 0.018 & 24 & 0.107 & 37 & 0.029 & 24 & 0.2547 \\
\hline k17 & 0.051 & 22 & 0.0231 & 27 & 0.0544 & 28 & 0.023 & 25 & 0.0998 & 36 & 0.034 & 25 & 0.2853 \\
\hline k26 & 0.0336 & 18 & 0.0241 & 28 & 0.0521 & 27 & 0.025 & 26 & 0.093 & 35 & 0.04 & 26 & 0.2678 \\
\hline k48 & 0.0134 & 12 & 0.0687 & 30 & 0.0109 & 15 & 0.069 & 28 & 0.0004 & 3 & 0.069 & 27 & 0.2314 \\
\hline k22 & 0.0647 & 23 & 0.0353 & 29 & 0.0655 & 29 & 0.031 & 27 & 0.225 & 38 & 0.084 & 28 & 0.5055 \\
\hline k20 & 0.104 & 26 & 0.0751 & 31 & 0.158 & 32 & 0.069 & 29 & 0.281 & 40 & 0.087 & 29 & 0.7741 \\
\hline k25 & 0.0756 & 24 & 0.0752 & 32 & 0.04 & 26 & 0.096 & 30 & 0.0053 & 10 & 0.098 & 30 & 0.3901 \\
\hline k50 & 0.179 & 30 & 0.153 & 34 & 0.0966 & 30 & 0.223 & 31 & 0.0018 & 7 & 0.225 & 31 & 0.8784 \\
\hline k24 & 0.0501 & 21 & 0.197 & 36 & 0.138 & 31 & 0.242 & 32 & 0.017 & 18 & 0.251 & 32 & 0.8951 \\
\hline k30 & 0.0763 & 25 & 0.24 & 38 & 0.193 & 33 & 0.294 & 33 & 0.0225 & 21 & 0.305 & 33 & 1.1308 \\
\hline k31 & 0.114 & 27 & 0.206 & 37 & 0.346 & 35 & 0.353 & 34 & 0.0217 & 20 & 0.363 & 34 & 1.4037 \\
\hline k49 & 0.0147 & 13 & 0.184 & 35 & 0.291 & 34 & 0.414 & 35 & 0.002 & 8 & 0.416 & 35 & 1.3217 \\
\hline k9 & 0.156 & 29 & 0.477 & 42 & 0.455 & 36 & 0.601 & 36 & 0.0539 & 32 & 0.622 & 36 & 2.3649 \\
\hline k10 & 0.225 & 32 & 0.41 & 40 & 0.808 & 39 & 0.731 & 37 & 0.0474 & 28 & 0.751 & 37 & 2.9724 \\
\hline k4 & 4.48 & 42 & 0.41 & 41 & 0.808 & 40 & 0.731 & 38 & 0.0474 & 29 & 0.751 & 38 & 7.2274 \\
\hline k38 & 1.61 & 39 & 1.73 & 45 & 1.51 & 43 & 1.03 & 40 & 2.41 & 44 & 0.962 & 39 & 9.252 \\
\hline k2 & 1.6 & 38 & 1.73 & 44 & 1.51 & 42 & 1.03 & 39 & 5.37 & 45 & 0.962 & 40 & 12.202 \\
\hline k28 & 0.478 & 35 & 4.87 & 46 & 0.745 & 38 & 1.89 & 42 & 0.313 & 41 & 1.89 & 41 & 10.186 \\
\hline k32 & 0.213 & 31 & 1.3 & 43 & 0.675 & 37 & 2.57 & 43 & 0.0146 & 16 & 2.57 & 42 & 7.3426 \\
\hline k1 & 6.96 & 43 & 7.06 & 48 & 5.54 & 45 & 4.18 & 45 & 12.3 & 47 & 3.75 & 43 & 39.79 \\
\hline k6 & 0.925 & 36 & 9.48 & 49 & 1.45 & 41 & 4.14 & 44 & 0.813 & 42 & 4.13 & 44 & 20.938 \\
\hline k44 & 0.0017 & 3 & 0.41 & 39 & 4.34 & 44 & 1.03 & 41 & 8.41 & 46 & 4.31 & 45 & 18.502 \\
\hline k15 & 36.9 & 50 & 0.0043 & 18 & 29.5 & 48 & 19.5 & 47 & 32.5 & 49 & 20.3 & 46 & 138.7 \\
\hline k3 & 1.52 & 37 & 13.5 & 50 & 11.9 & 46 & 21.3 & 48 & 0.275 & 39 & 21.5 & 47 & 69.995 \\
\hline k13 & 36.9 & 51 & 0.0043 & 19 & 29.5 & 49 & 18.7 & 46 & 84.7 & 50 & 24.1 & 48 & 193.9 \\
\hline k11 & 36.8 & 49 & 0.0043 & 17 & 32 & 50 & 25.5 & 49 & 32.2 & 48 & 25 & 49 & 151.5 \\
\hline ML & 36.2 & 48 & 6.84 & 47 & 33.008 & 51 & 28.004 & 50 & 97.009 & 51 & 28.045 & 50 & 229.11 \\
\hline k39 & 14.7 & 47 & 13.7 & 51 & 13.4 & 47 & 171 & 51 & 2.27 & 43 & 169 & 51 & 384.07 \\
\hline
\end{tabular}


Table 3. Ranked Mean Square Error of estimators for $p=4$ and $\beta=1$ at varying correlation levels and sample sizes.

\begin{tabular}{|c|c|c|c|c|c|c|c|c|c|c|c|c|c|}
\hline \multirow[b]{2}{*}{ Estimators } & \multicolumn{4}{|l|}{$\rho=0.85$} & \multicolumn{4}{|l|}{$\rho=0.90$} & \multicolumn{5}{|l|}{$\rho=0.99$} \\
\hline & $\begin{array}{l}\text { Sample } \\
50(0.85) \\
\end{array}$ & R1 & $\begin{array}{l}\text { Sample } \\
200(0.85) \\
\end{array}$ & $\mathbf{R 2}$ & $\begin{array}{l}\text { Sample } \\
50(0.90)\end{array}$ & $\mathbf{R 3}$ & $\begin{array}{l}\text { Sample } \\
200(0.90) \\
\end{array}$ & R4 & $\begin{array}{l}\text { Sample } \\
50(0.99) \\
\end{array}$ & R5 & $\begin{array}{l}\text { Sample } \\
200(0.99) \\
\end{array}$ & R6 & Total MSE \\
\hline k14 & 0.0005 & 3 & 0 & 3 & 0.0003 & 3 & 0 & 3 & 0 & 1 & 0 & 1 & 0.0008 \\
\hline k12 & 0.0006 & 4 & 0 & 4 & 0.0003 & 4 & 0 & 4 & 0 & 2 & 0 & 2 & 0.0009 \\
\hline k16 & 0.0006 & 5 & 0 & 5 & 0.0003 & 5 & 0 & 5 & 0 & 3 & 0 & 3 & 0.0009 \\
\hline k36 & 0 & 1 & 0 & 1 & 0 & 1 & 0 & 1 & 0.0001 & 4 & 0 & 4 & 0.0001 \\
\hline k37 & 0.0001 & 2 & 0 & 2 & 0 & 2 & 0 & 2 & 0.0004 & 5 & 0 & 5 & 0.0005 \\
\hline k34 & 0.0046 & 6 & 0 & 6 & 0.0009 & 6 & 0 & 6 & 0.0067 & 10 & 0 & 6 & 0.0122 \\
\hline k45 & 0.0078 & 7 & 0 & 7 & 0.0014 & 7 & 0 & 7 & 0.0179 & 17 & 0 & 7 & 0.0271 \\
\hline k46 & 0.0164 & 9 & 0 & 9 & 0.0069 & 12 & 0 & 11 & 0.0179 & 18 & 0 & 8 & 0.0412 \\
\hline k47 & 0.0223 & 12 & 0 & 12 & 0.0116 & 16 & 0 & 12 & 0.0263 & 22 & 0 & 9 & 0.0602 \\
\hline k35 & 0.0185 & 10 & 0 & 10 & 0.0034 & 10 & 0 & 10 & 0.0264 & 23 & 0 & 10 & 0.0483 \\
\hline k43 & 0.0145 & 8 & 0 & 8 & 0.0014 & 8 & 0 & 8 & 0.251 & 27 & 0 & 11 & 0.2669 \\
\hline k42 & 0.0199 & 11 & 0 & 11 & 0.0032 & 9 & 0 & 9 & 0.251 & 28 & 0 & 12 & 0.2741 \\
\hline k33 & 0.0254 & 13 & 0.001 & 13 & 0.021 & 21 & 0.001 & 14 & 0.0262 & 21 & 0.001 & 13 & 0.0756 \\
\hline k41 & 0.0621 & 19 & 0.001 & 14 & 0.0107 & 15 & 0.001 & 13 & 0.252 & 29 & 0.001 & 14 & 0.3278 \\
\hline k7 & 0.028 & 14 & 0.004 & 15 & 0.009 & 13 & 0.003 & 15 & 0.0043 & 8 & 0.003 & 15 & 0.0513 \\
\hline k8 & 0.0323 & 15 & 0.004 & 16 & 0.0091 & 14 & 0.004 & 16 & 0.0041 & 7 & 0.004 & 16 & 0.0575 \\
\hline k40 & 0.058 & 18 & 0.005 & 17 & 0.0059 & 11 & 0.005 & 17 & 0.0034 & 6 & 0.005 & 17 & 0.0823 \\
\hline k21 & 0.0513 & 16 & 0.006 & 18 & 0.0144 & 17 & 0.006 & 18 & 0.0059 & 9 & 0.006 & 18 & 0.0896 \\
\hline k29 & 0.0574 & 17 & 0.007 & 19 & 0.0169 & 18 & 0.007 & 19 & 0.008 & 12 & 0.007 & 19 & 0.1033 \\
\hline k27 & 0.066 & 20 & 0.008 & 20 & 0.0172 & 19 & 0.007 & 20 & 0.0078 & 11 & 0.008 & 20 & 0.114 \\
\hline k18 & 0.0685 & 21 & 0.009 & 21 & 0.0202 & 20 & 0.008 & 21 & 0.0095 & 13 & 0.008 & 21 & 0.1232 \\
\hline $\mathrm{k} 23$ & 0.0909 & 23 & 0.01 & 22 & 0.0224 & 22 & 0.009 & 22 & 0.0113 & 14 & 0.009 & 22 & 0.1526 \\
\hline k5 & 0.0715 & 22 & 0.012 & 23 & 0.0285 & 23 & 0.012 & 23 & 0.0113 & 15 & 0.012 & 23 & 0.1473 \\
\hline k19 & 0.132 & 26 & 0.019 & 24 & 0.0356 & 24 & 0.019 & 24 & 0.0167 & 16 & 0.019 & 24 & 0.2413 \\
\hline k17 & 0.163 & 29 & 0.025 & 25 & 0.0549 & 26 & 0.024 & 25 & 0.0228 & 20 & 0.024 & 25 & 0.3137 \\
\hline k26 & 0.15 & 28 & 0.026 & 26 & 0.0546 & 25 & 0.025 & 26 & 0.0212 & 19 & 0.025 & 26 & 0.3018 \\
\hline k22 & 0.257 & 32 & 0.03 & 27 & 0.0624 & 27 & 0.027 & 27 & 0.0328 & 24 & 0.027 & 27 & 0.4362 \\
\hline k20 & 0.419 & 35 & 0.071 & 28 & 0.158 & 29 & 0.068 & 28 & 0.0654 & 25 & 0.067 & 28 & 0.8484 \\
\hline $\mathrm{k} 25$ & 0.102 & 24 & 0.116 & 30 & 0.146 & 28 & 0.124 & 29 & 0.206 & 26 & 0.123 & 29 & 0.817 \\
\hline k48 & 0.106 & 25 & 0.11 & 29 & 0.178 & 30 & 0.135 & 30 & 0.598 & 33 & 0.127 & 30 & 1.254 \\
\hline k24 & 0.168 & 30 & 0.228 & 32 & 0.193 & 31 & 0.213 & 31 & 0.274 & 30 & 0.207 & 31 & 1.283 \\
\hline k50 & 0.143 & 27 & 0.223 & 31 & 0.21 & 32 & 0.217 & 32 & 0.721 & 34 & 0.208 & 32 & 1.722 \\
\hline k30 & 0.256 & 31 & 0.299 & 33 & 0.307 & 33 & 0.288 & 33 & 0.434 & 31 & 0.283 & 33 & 1.867 \\
\hline k31 & 0.343 & 34 & 0.36 & 34 & 0.39 & 35 & 0.339 & 34 & 0.451 & 32 & 0.335 & 34 & 2.218 \\
\hline k49 & 0.257 & 33 & 0.411 & 35 & 0.33 & 34 & 0.377 & 35 & 0.869 & 35 & 0.369 & 35 & 2.613 \\
\hline k9 & 0.531 & 36 & 0.612 & 36 & 0.673 & 37 & 0.605 & 36 & 0.965 & 36 & 0.593 & 36 & 3.979 \\
\hline k4 & 0.738 & 39 & 0.747 & 37 & 0.885 & 39 & 0.716 & 37 & 1.01 & 37 & 0.705 & 37 & 4.801 \\
\hline k10 & 0.738 & 40 & 0.747 & 38 & 0.885 & 40 & 0.716 & 38 & 1.01 & 38 & 0.705 & 38 & 4.801 \\
\hline k2 & 4.33 & 43 & 1.38 & 40 & 2.42 & 43 & 1.7 & 41 & 3.34 & 40 & 1.51 & 39 & 14.68 \\
\hline k38 & 1.89 & 42 & 1.38 & 39 & 2.42 & 42 & 1.7 & 40 & 7.4 & 44 & 1.51 & 40 & 16.3 \\
\hline k28 & 0.673 & 38 & 1.85 & 41 & 0.648 & 36 & 1.7 & 39 & 3.42 & 41 & 1.64 & 41 & 9.931 \\
\hline k32 & 0.666 & 37 & 2.36 & 42 & 0.844 & 38 & 2.24 & 42 & 2.7 & 39 & 2.17 & 42 & 10.98 \\
\hline k6 & 1.34 & 41 & 4.06 & 43 & 1.28 & 41 & 3.71 & 43 & 6.66 & 43 & 3.58 & 43 & 20.63 \\
\hline k1 & 6.73 & 44 & 5.5 & 44 & 10.1 & 44 & 7.78 & 44 & 15.2 & 45 & 6.74 & 44 & 52.05 \\
\hline k3 & 13.8 & 46 & 21.4 & 48 & 21.4 & 46 & 21.8 & 45 & 39.5 & 47 & 20.8 & 45 & 138.7 \\
\hline ML & 79.0084 & 51 & 23.005 & 49 & 54.0072 & 51 & 23.005 & 46 & 64.0058 & 51 & 42.005 & 46 & 285.0364 \\
\hline k13 & 66.8 & 50 & 18.8 & 46 & 37.9 & 48 & 86.6 & 47 & 58.4 & 49 & 47.7 & 47 & 316.2 \\
\hline k15 & 32 & 49 & 20.4 & 47 & 37.9 & 49 & 88.6 & 48 & 58.4 & 50 & 49.1 & 48 & 286.4 \\
\hline k11 & 30.9 & 48 & 25.6 & 50 & 38.6 & 50 & 88.7 & 49 & 58.2 & 48 & 50.7 & 49 & 292.7 \\
\hline k44 & 17.8 & 47 & 12.9 & 45 & 36.1 & 47 & 96.4 & 50 & 5.81 & 42 & 55.1 & 50 & 224.11 \\
\hline k39 & 11 & 45 & 168 & 51 & 13.2 & 45 & 154 & 51 & 18.9 & 46 & 147 & 51 & 512.1 \\
\hline
\end{tabular}


Table 4. Ranked Mean Square Error of estimators for $p=8$ and $\beta=-1$ at varying correlation levels and sample sizes.

\begin{tabular}{|c|c|c|c|c|c|c|c|c|c|c|c|c|c|}
\hline \multirow[b]{2}{*}{ Estimators } & \multicolumn{4}{|l|}{$\rho=0.85$} & \multicolumn{4}{|l|}{$\rho=0.90$} & \multicolumn{5}{|l|}{$\rho=0.99$} \\
\hline & $\begin{array}{l}\text { Sample } \\
50(0.85)\end{array}$ & R1 & $\begin{array}{l}\text { Sample } \\
200(0.85)\end{array}$ & $\mathbf{R 2}$ & $\begin{array}{l}\text { Sample } \\
50(0.90)\end{array}$ & $\mathbf{R 3}$ & $\begin{array}{l}\text { Sample } \\
200(0.90)\end{array}$ & R4 & $\begin{array}{l}\text { Sample } \\
50(0.99)\end{array}$ & R5 & $\begin{array}{l}\text { Sample } \\
200(0.99)\end{array}$ & R6 & Total MSE \\
\hline k36 & 0.0001 & 4 & 0 & 1 & 0 & 1 & 0 & 1 & 0.0001 & 4 & 0.0001 & 1 & 0.0003 \\
\hline k37 & 0.0004 & 5 & 0.0001 & 2 & 0.0001 & 2 & 0 & 2 & 0.0004 & 5 & 0.0003 & 2 & 0.0013 \\
\hline k12 & 0 & 1 & 0.0004 & 3 & 0.0004 & 3 & 0 & 3 & 0 & 1 & 0.0004 & 3 & 0.0012 \\
\hline k14 & 0 & 2 & 0.0004 & 4 & 0.0004 & 4 & 0 & 4 & 0 & 2 & 0.0004 & 4 & 0.0012 \\
\hline k16 & 0 & 3 & 0.0004 & 5 & 0.0004 & 5 & 0 & 5 & 0 & 3 & 0.0004 & 5 & 0.0012 \\
\hline k34 & 0.0067 & 10 & 0.002 & 6 & 0.0022 & 7 & 0 & 7 & 0.0064 & 10 & 0.0052 & 6 & 0.0225 \\
\hline k40 & 0.0028 & 6 & 0.0048 & 7 & 0.0048 & 9 & 0.005 & 17 & 0.0034 & 6 & 0.006 & 7 & 0.0268 \\
\hline k8 & 0.0037 & 8 & 0.0095 & 12 & 0.0094 & 14 & 0.004 & 16 & 0.0043 & 7 & 0.0119 & 8 & 0.0428 \\
\hline k7 & 0.0037 & 7 & 0.0095 & 11 & 0.0093 & 13 & 0.004 & 15 & 0.0044 & 8 & 0.0119 & 9 & 0.0428 \\
\hline k46 & 0.0183 & 19 & 0.0078 & 9 & 0.007 & 11 & 0 & 8 & 0.0176 & 18 & 0.0126 & 10 & 0.0633 \\
\hline k45 & 0.0183 & 18 & 0.0051 & 8 & 0.0017 & 6 & 0 & 6 & 0.0175 & 16 & 0.014 & 11 & 0.0566 \\
\hline k21 & 0.0049 & 9 & 0.014 & 14 & 0.0138 & 17 & 0.006 & 18 & 0.0059 & 9 & 0.0182 & 12 & 0.0628 \\
\hline k47 & 0.0273 & 23 & 0.0122 & 13 & 0.0115 & 15 & 0 & 10 & 0.0259 & 23 & 0.0183 & 13 & 0.0952 \\
\hline k33 & 0.0271 & 22 & 0.0209 & 17 & 0.0213 & 21 & 0.001 & 11 & 0.0258 & 22 & 0.0196 & 14 & 0.1157 \\
\hline k35 & 0.0265 & 21 & 0.0079 & 10 & 0.0087 & 12 & 0 & 9 & 0.0252 & 21 & 0.0206 & 15 & 0.0889 \\
\hline k29 & 0.0071 & 11 & 0.0179 & 15 & 0.0176 & 18 & 0.008 & 19 & 0.0083 & 12 & 0.0224 & 16 & 0.0813 \\
\hline k27 & 0.0072 & 12 & 0.0183 & 16 & 0.0179 & 19 & 0.008 & 20 & 0.0081 & 11 & 0.0226 & 17 & 0.0821 \\
\hline k18 & 0.0083 & 13 & 0.0214 & 18 & 0.021 & 20 & 0.009 & 21 & 0.0098 & 13 & 0.0264 & 18 & 0.0959 \\
\hline k23 & 0.0101 & 15 & 0.0225 & 19 & 0.0228 & 22 & 0.011 & 22 & 0.0119 & 15 & 0.0269 & 19 & 0.1052 \\
\hline k5 & 0.0089 & 14 & 0.0278 & 20 & 0.0278 & 23 & 0.012 & 23 & 0.0111 & 14 & 0.034 & 20 & 0.1216 \\
\hline k19 & 0.0158 & 16 & 0.0376 & 21 & 0.0367 & 24 & 0.02 & 24 & 0.0175 & 17 & 0.0461 & 21 & 0.1737 \\
\hline k26 & 0.0166 & 17 & 0.0534 & 22 & 0.0535 & 25 & 0.025 & 26 & 0.0208 & 19 & 0.0657 & 22 & 0.235 \\
\hline k17 & 0.0186 & 20 & 0.0557 & 23 & 0.0549 & 26 & 0.024 & 25 & 0.0226 & 20 & 0.0686 & 23 & 0.2444 \\
\hline k22 & 0.0291 & 24 & 0.065 & 24 & 0.0663 & 27 & 0.033 & 27 & 0.0347 & 24 & 0.0688 & 24 & 0.2969 \\
\hline $\mathrm{k} 25$ & 0.209 & 26 & 0.0725 & 28 & 0.0778 & 28 & 0.128 & 29 & 0.203 & 26 & 0.185 & 25 & 0.8753 \\
\hline k20 & 0.048 & 25 & 0.162 & 29 & 0.161 & 29 & 0.069 & 28 & 0.0634 & 25 & 0.188 & 26 & 0.6914 \\
\hline k41 & 0.253 & 29 & 0.0698 & 26 & 0.0126 & 16 & 0.003 & 14 & 0.245 & 29 & 0.199 & 27 & 0.7824 \\
\hline k42 & 0.252 & 28 & 0.0698 & 25 & 0.0051 & 10 & 0.002 & 13 & 0.245 & 28 & 0.203 & 28 & 0.7769 \\
\hline k43 & 0.252 & 27 & 0.07 & 27 & 0.0038 & 8 & 0.002 & 12 & 0.245 & 27 & 0.204 & 29 & 0.7768 \\
\hline k24 & 1.06 & 30 & 0.187 & 30 & 0.207 & 30 & 0.48 & 30 & 0.307 & 30 & 0.405 & 30 & 2.646 \\
\hline k32 & 1.68 & 31 & 0.615 & 37 & 0.655 & 36 & 2.48 & 39 & 1.75 & 39 & 0.605 & 31 & 7.785 \\
\hline k30 & 1.72 & 32 & 0.289 & 32 & 0.321 & 31 & 0.762 & 31 & 0.493 & 31 & 0.67 & 32 & 4.255 \\
\hline k48 & 8.6 & 39 & 0.267 & 31 & 0.36 & 32 & 2.15 & 38 & 0.564 & 33 & 0.712 & 33 & 12.653 \\
\hline k31 & 1.79 & 33 & 0.389 & 34 & 0.426 & 33 & 0.815 & 32 & 0.517 & 32 & 0.718 & 34 & 4.655 \\
\hline k50 & 10.3 & 40 & 0.326 & 33 & 0.43 & 34 & 2.57 & 40 & 0.678 & 34 & 0.839 & 35 & 15.143 \\
\hline k28 & 3.28 & 34 & 0.748 & 38 & 0.775 & 38 & 1.97 & 36 & 3.41 & 40 & 0.949 & 36 & 11.132 \\
\hline k49 & 11.9 & 41 & 0.447 & 35 & 0.559 & 35 & 3.07 & 41 & 0.821 & 35 & 0.998 & 37 & 17.795 \\
\hline k9 & 3.83 & 35 & 0.606 & 36 & 0.732 & 37 & 1.64 & 33 & 1.11 & 36 & 1.35 & 38 & 9.268 \\
\hline k4 & 3.97 & 36 & 0.85 & 39 & 0.979 & 39 & 1.76 & 34 & 1.16 & 37 & 1.47 & 39 & 10.189 \\
\hline k10 & 3.97 & 37 & 0.85 & 40 & 0.979 & 40 & 1.76 & 35 & 1.16 & 38 & 1.47 & 40 & 10.189 \\
\hline k6 & 6.83 & 38 & 1.46 & 41 & 1.56 & 41 & 4.32 & 42 & 6.66 & 43 & 1.93 & 41 & 22.76 \\
\hline k44 & 19.7 & 43 & 4.19 & 44 & 8.4 & 45 & 2.13 & 37 & 9.6 & 44 & 3.25 & 42 & 47.27 \\
\hline k2 & 49.5 & 47 & 2.06 & 42 & 2.62 & 42 & 12.1 & 44 & 4.51 & 41 & 3.52 & 43 & 74.31 \\
\hline k38 & 49.5 & 48 & 2.06 & 43 & 2.62 & 43 & 12.1 & 45 & 4.51 & 42 & 3.52 & 44 & 74.31 \\
\hline k11 & 72 & 50 & 32.6 & 50 & 27.1 & 50 & 28.7 & 48 & 68.7 & 49 & 10.7 & 45 & 239.8 \\
\hline k3 & 48.9 & 46 & 23.9 & 47 & 12.1 & 47 & 33.6 & 50 & 46.8 & 47 & 11.2 & 46 & 176.5 \\
\hline k15 & 17.2 & 42 & 28.3 & 48 & 11.5 & 46 & 23.9 & 47 & 69.3 & 50 & 11.9 & 47 & 162.1 \\
\hline k39 & 21.3 & 44 & 13.7 & 46 & 14.5 & 48 & 166 & 51 & 21.7 & 46 & 13.5 & 48 & 250.7 \\
\hline k1 & 24.2 & 45 & 7.94 & 45 & 6.74 & 44 & 7.37 & 43 & 17.5 & 45 & 13.9 & 49 & 77.65 \\
\hline k13 & 71.8 & 49 & 28.3 & 49 & 21.1 & 49 & 20.9 & 46 & 68.7 & 48 & 20 & 50 & 230.8 \\
\hline ML & 92.1 & 51 & 49.2 & 51 & 41.4 & 51 & 28.8 & 49 & 83.8 & 51 & 36.3 & 51 & 331.6 \\
\hline
\end{tabular}


Table 5. Ranked Mean Square Error of estimators for $p=8$ and $\beta=0$ at varying correlation levels and sample sizes.

\begin{tabular}{|c|c|c|c|c|c|c|c|c|c|c|c|c|c|}
\hline \multirow[b]{2}{*}{ Estimators } & \multicolumn{4}{|l|}{$\rho=0.85$} & \multicolumn{4}{|l|}{$\rho=0.90$} & \multicolumn{5}{|l|}{$\rho=0.99$} \\
\hline & $\begin{array}{l}\text { Sample } \\
50(0.85)\end{array}$ & R1 & $\begin{array}{l}\text { Sample } \\
200(0.85)\end{array}$ & R2 & $\begin{array}{l}\text { Sample } \\
50(0.90)\end{array}$ & R3 & $\begin{array}{l}\text { Sample } \\
200(0.90)\end{array}$ & R4 & $\begin{array}{l}\text { Sample } \\
50(0.99)\end{array}$ & R5 & $\begin{array}{l}\text { Sample } \\
200(0.99)\end{array}$ & R6 & Total MSE \\
\hline k36 & 0 & 1 & 0 & 1 & 0.0001 & 1 & 0 & 1 & 0.0001 & 1 & 0 & 1 & 0.0002 \\
\hline k37 & 0.0001 & 2 & 0 & 2 & 0.0002 & 2 & 0.0001 & 2 & 0.0002 & 2 & 0 & 2 & 0.0006 \\
\hline k14 & 0.0003 & 3 & 0 & 3 & 0.0004 & 3 & 0.0004 & 3 & 0.0006 & 4 & 0 & 3 & 0.0017 \\
\hline k16 & 0.0003 & 4 & 0 & 4 & 0.0004 & 4 & 0.0004 & 4 & 0.0008 & 5 & 0 & 4 & 0.0019 \\
\hline k12 & 0.0004 & 5 & 0 & 5 & 0.0004 & 5 & 0.0004 & 5 & 0.0009 & 6 & 0 & 5 & 0.0021 \\
\hline k34 & 0.0022 & 7 & 0 & 7 & 0.0039 & 7 & 0.0022 & 6 & 0.0042 & 9 & 0 & 6 & 0.0125 \\
\hline $\mathrm{k} 45$ & 0.0017 & 6 & 0 & 6 & 0.0019 & 6 & 0.0057 & 8 & 0.0089 & 13 & 0 & 7 & 0.0182 \\
\hline k35 & 0.0087 & 12 & 0 & 9 & 0.0155 & 18 & 0.0087 & 10 & 0.0165 & 15 & 0 & 8 & 0.0494 \\
\hline k46 & 0.0067 & 11 & 0 & 8 & 0.007 & 11 & 0.0082 & 9 & 0.0069 & 10 & 0.002 & 9 & 0.0308 \\
\hline k47 & 0.0109 & 15 & 0 & 10 & 0.0113 & 15 & 0.0126 & 14 & 0.007 & 11 & 0.002 & 10 & 0.0438 \\
\hline k42 & 0.0048 & 10 & 0.002 & 13 & 0.0066 & 10 & 0.0779 & 28 & 0.0168 & 16 & 0.002 & 11 & 0.1101 \\
\hline $\mathrm{k} 43$ & 0.0037 & 8 & 0.002 & 12 & 0.006 & 9 & 0.0781 & 29 & 0.0154 & 14 & 0.003 & 12 & 0.1082 \\
\hline k33 & 0.0201 & 21 & 0.001 & 11 & 0.0212 & 22 & 0.0209 & 18 & 0.0249 & 22 & 0.003 & 13 & 0.0911 \\
\hline k41 & 0.0118 & 17 & 0.002 & 14 & 0.0123 & 16 & 0.0778 & 27 & 0.0584 & 30 & 0.004 & 14 & 0.1663 \\
\hline $\mathrm{k} 40$ & 0.0048 & 9 & 0.005 & 17 & 0.0053 & 8 & 0.0049 & 7 & 0.0629 & 32 & 0.005 & 15 & 0.0879 \\
\hline k18 & 0.0208 & 22 & 0.008 & 21 & 0.0206 & 21 & 0.0218 & 19 & 0.0586 & 31 & 0.009 & 16 & 0.1388 \\
\hline $\mathrm{k} 7$ & 0.0092 & 13 & 0.003 & 15 & 0.0091 & 12 & 0.0096 & 11 & 0.022 & 19 & 0.013 & 17 & 0.0659 \\
\hline k8 & 0.0093 & 14 & 0.004 & 16 & 0.0097 & 13 & 0.0096 & 12 & 0.0229 & 20 & 0.015 & 18 & 0.0705 \\
\hline k21 & 0.0134 & 18 & 0.006 & 18 & 0.0135 & 17 & 0.014 & 15 & 0.0313 & 23 & 0.018 & 19 & 0.0962 \\
\hline k19 & 0.0365 & 25 & 0.019 & 24 & 0.0364 & 25 & 0.0383 & 22 & 0.124 & 36 & 0.018 & 20 & 0.2722 \\
\hline k29 & 0.0173 & 19 & 0.007 & 19 & 0.0176 & 19 & 0.0182 & 16 & 0.0449 & 24 & 0.024 & 21 & 0.129 \\
\hline k27 & 0.0178 & 20 & 0.007 & 20 & 0.0195 & 20 & 0.0185 & 17 & 0.045 & 25 & 0.028 & 22 & 0.1358 \\
\hline k17 & 0.0535 & 28 & 0.024 & 25 & 0.0532 & 27 & 0.0562 & 25 & 0.113 & 35 & 0.031 & 23 & 0.3309 \\
\hline k5 & 0.0266 & 24 & 0.012 & 23 & 0.027 & 24 & 0.0273 & 21 & 0.0492 & 28 & 0.034 & 24 & 0.1761 \\
\hline $\mathrm{k} 23$ & 0.0226 & 23 & 0.011 & 22 & 0.0268 & 23 & 0.0228 & 20 & 0.0704 & 33 & 0.042 & 25 & 0.1956 \\
\hline k26 & 0.0509 & 27 & 0.025 & 26 & 0.0535 & 28 & 0.0521 & 24 & 0.102 & 34 & 0.064 & 26 & 0.3475 \\
\hline $\mathrm{k} 48$ & 0.0111 & 16 & 0.093 & 29 & 0.0108 & 14 & 0.0107 & 13 & 0.0004 & 3 & 0.07 & 27 & 0.196 \\
\hline k25 & 0.0418 & 26 & 0.105 & 30 & 0.0409 & 26 & 0.0439 & 23 & 0.0079 & 12 & 0.099 & 28 & 0.3385 \\
\hline $\mathrm{k} 20$ & 0.155 & 32 & 0.069 & 28 & 0.155 & 32 & 0.161 & 32 & 0.287 & 40 & 0.106 & 29 & 0.933 \\
\hline k22 & 0.0774 & 29 & 0.049 & 27 & 0.123 & 30 & 0.0685 & 26 & 0.274 & 39 & 0.164 & 30 & 0.7559 \\
\hline $\mathrm{k} 50$ & 0.101 & 30 & 0.218 & 31 & 0.0944 & 29 & 0.0932 & 30 & 0.002 & 7 & 0.23 & 31 & 0.7386 \\
\hline k24 & 0.141 & 31 & 0.233 & 32 & 0.138 & 31 & 0.133 & 31 & 0.0171 & 17 & 0.271 & 32 & 0.9331 \\
\hline k30 & 0.2 & 33 & 0.29 & 33 & 0.191 & 33 & 0.187 & 33 & 0.0244 & 21 & 0.33 & 33 & 1.2224 \\
\hline k31 & 0.361 & 35 & 0.349 & 34 & 0.342 & 35 & 0.331 & 35 & 0.0217 & 18 & 0.39 & 34 & 1.7947 \\
\hline k49 & 0.304 & 34 & 0.402 & 35 & 0.283 & 34 & 0.275 & 34 & 0.002 & 8 & 0.427 & 35 & 1.693 \\
\hline k9 & 0.475 & 36 & 0.596 & 36 & 0.446 & 36 & 0.437 & 36 & 0.0577 & 29 & 0.677 & 36 & 2.6887 \\
\hline k4 & 0.846 & 39 & 0.725 & 37 & 0.791 & 39 & 0.766 & 38 & 0.0475 & 26 & 0.81 & 37 & 3.9855 \\
\hline $\mathrm{k} 10$ & 0.846 & 40 & 0.725 & 38 & 0.791 & 40 & 0.766 & 39 & 0.0475 & 27 & 0.81 & 38 & 3.9855 \\
\hline k38 & 1.5 & 42 & 1.19 & 39 & 6.97 & 43 & 1.49 & 40 & 1.56 & 41 & 0.989 & 39 & 13.699 \\
\hline k2 & 1.5 & 43 & 1.19 & 40 & 6.97 & 44 & 1.49 & 41 & 5.76 & 44 & 0.989 & 40 & 17.899 \\
\hline $\mathrm{k} 28$ & 0.732 & 38 & 1.82 & 41 & 0.724 & 38 & 4.67 & 43 & 6.11 & 45 & 1.79 & 41 & 15.846 \\
\hline k32 & 0.662 & 37 & 2.42 & 42 & 0.627 & 37 & 0.63 & 37 & 0.256 & 38 & 2.48 & 42 & 7.075 \\
\hline k1 & 5.48 & 44 & 4.89 & 44 & 25.4 & 47 & 5.53 & 44 & 5.19 & 43 & 3.87 & 43 & 50.36 \\
\hline k6 & 1.43 & 41 & 3.97 & 43 & 1.4 & 41 & 9.28 & 45 & 13.2 & 47 & 3.88 & 44 & 33.16 \\
\hline $\mathrm{k} 44$ & 9.06 & 45 & 69.4 & 50 & 4.2 & 42 & 4.24 & 42 & 8.28 & 46 & 4 & 45 & 99.18 \\
\hline k13 & 29.5 & 49 & 63.5 & 47 & 46.3 & 48 & 28.8 & 48 & 88.2 & 51 & 17.4 & 46 & 273.7 \\
\hline k15 & 29.5 & 48 & 64.8 & 48 & 46.3 & 49 & 28.8 & 49 & 18.7 & 49 & 18.2 & 47 & 206.3 \\
\hline ML & 48.1 & 51 & 28.4 & 46 & 84.1 & 51 & 30.3 & 50 & 19.1 & 50 & 20.7 & 48 & 230.7 \\
\hline k3 & 12.5 & 47 & 21 & 45 & 11.6 & 45 & 11.3 & 46 & 0.249 & 37 & 22.1 & 49 & 78.749 \\
\hline k11 & 31.1 & 50 & 66.1 & 49 & 47.2 & 50 & 32.1 & 51 & 17 & 48 & 24.2 & 50 & 217.7 \\
\hline k39 & 12.4 & 46 & 163 & 51 & 12.7 & 46 & 13.4 & 47 & 2.99 & 42 & 151 & 51 & 355.49 \\
\hline
\end{tabular}


Table 6. Ranked Mean Square Error of estimators for $p=8$ and $\beta=1$ at varying correlation levels and sample sizes.

\begin{tabular}{|c|c|c|c|c|c|c|c|c|c|c|c|c|c|}
\hline \multirow[b]{2}{*}{ Estimators } & \multicolumn{4}{|l|}{$\rho=0.85$} & \multicolumn{4}{|l|}{$\rho=0.90$} & \multicolumn{5}{|l|}{$\rho=0.99$} \\
\hline & $\begin{array}{l}\text { Sample } \\
50(0.85)\end{array}$ & R1 & $\begin{array}{l}\text { Sample } \\
200(0.85)\end{array}$ & $\mathbf{R} 2$ & $\begin{array}{l}\text { Sample } \\
50(0.90)\end{array}$ & $\mathbf{R 3}$ & $\begin{array}{l}\text { Sample } \\
200(0.90)\end{array}$ & R4 & $\begin{array}{l}\text { Sample } \\
50(0.99)\end{array}$ & R5 & $\begin{array}{l}\text { Sample } \\
200(0.99)\end{array}$ & R6 & Total MSE \\
\hline k36 & 0 & 1 & 0.0001 & 1 & 0.0001 & 1 & 0.0001 & 1 & 0 & 1 & 0 & 1 & 0.0003 \\
\hline k37 & 0 & 2 & 0.0002 & 2 & 0.0003 & 2 & 0.0004 & 2 & 0 & 2 & 0.0001 & 2 & 0.001 \\
\hline k12 & 0 & 3 & 0.0004 & 3 & 0.0004 & 3 & 0.0004 & 3 & 0 & 3 & 0.0004 & 3 & 0.0016 \\
\hline k14 & 0 & 4 & 0.0004 & 4 & 0.0004 & 4 & 0.0004 & 4 & 0 & 4 & 0.0004 & 4 & 0.0016 \\
\hline k16 & 0 & 5 & 0.0004 & 5 & 0.0004 & 5 & 0.0004 & 5 & 0 & 5 & 0.0004 & 5 & 0.0016 \\
\hline k34 & 0 & 6 & 0.0032 & 6 & 0.0052 & 7 & 0.0061 & 7 & 0 & 6 & 0.0022 & 6 & 0.0167 \\
\hline $\mathrm{k} 40$ & 0.005 & 10 & 0.0051 & 7 & 0.0057 & 8 & 0.006 & 6 & 0.005 & 10 & 0.0048 & 7 & 0.0316 \\
\hline $\mathrm{k} 45$ & 0.013 & 17 & 0.0074 & 8 & 0.0023 & 6 & 0.0163 & 11 & 0.017 & 18 & 0.0057 & 8 & 0.0617 \\
\hline k46 & 0.013 & 18 & 0.009 & 9 & 0.008 & 9 & 0.014 & 10 & 0.017 & 17 & 0.008 & 9 & 0.069 \\
\hline k35 & 0 & 7 & 0.0125 & 12 & 0.0206 & 19 & 0.024 & 17 & 0 & 7 & 0.0087 & 10 & 0.0658 \\
\hline k7 & 0.004 & 8 & 0.0098 & 10 & 0.0095 & 11 & 0.0115 & 8 & 0.004 & 8 & 0.0095 & 11 & 0.0483 \\
\hline k8 & 0.004 & 9 & 0.0098 & 11 & 0.0095 & 12 & 0.0116 & 9 & 0.004 & 9 & 0.0095 & 12 & 0.0484 \\
\hline k47 & 0.019 & 19 & 0.0138 & 13 & 0.0138 & 14 & 0.0201 & 14 & 0.026 & 23 & 0.0124 & 13 & 0.1051 \\
\hline $\mathrm{k} 21$ & 0.006 & 11 & 0.0148 & 14 & 0.0153 & 15 & 0.0178 & 12 & 0.006 & 11 & 0.0141 & 14 & 0.074 \\
\hline k29 & 0.007 & 12 & 0.0186 & 15 & 0.0179 & 17 & 0.0218 & 15 & 0.007 & 12 & 0.018 & 15 & 0.0903 \\
\hline k27 & 0.008 & 13 & 0.0187 & 16 & 0.018 & 18 & 0.0219 & 16 & 0.008 & 13 & 0.018 & 16 & 0.0926 \\
\hline k33 & 0.019 & 20 & 0.0214 & 17 & 0.0238 & 21 & 0.0178 & 13 & 0.026 & 22 & 0.0208 & 17 & 0.1288 \\
\hline k18 & 0.009 & 14 & 0.0221 & 18 & 0.0211 & 20 & 0.0256 & 18 & 0.008 & 14 & 0.0214 & 18 & 0.1072 \\
\hline $\mathrm{k} 23$ & 0.01 & 15 & 0.0237 & 19 & 0.0242 & 22 & 0.0263 & 19 & 0.01 & 15 & 0.0231 & 19 & 0.1173 \\
\hline k5 & 0.012 & 16 & 0.0293 & 20 & 0.0307 & 23 & 0.033 & 20 & 0.012 & 16 & 0.0285 & 20 & 0.1455 \\
\hline k19 & 0.02 & 21 & 0.038 & 21 & 0.037 & 24 & 0.0448 & 21 & 0.02 & 19 & 0.0362 & 21 & 0.196 \\
\hline k26 & 0.026 & 23 & 0.0564 & 22 & 0.0597 & 26 & 0.0632 & 22 & 0.025 & 21 & 0.055 & 22 & 0.2853 \\
\hline k17 & 0.025 & 22 & 0.0575 & 23 & 0.0575 & 25 & 0.0665 & 23 & 0.024 & 20 & 0.0554 & 23 & 0.2859 \\
\hline $\mathrm{k} 22$ & 0.029 & 24 & 0.067 & 24 & 0.0677 & 27 & 0.0665 & 24 & 0.028 & 24 & 0.0663 & 24 & 0.3245 \\
\hline k41 & 0.17 & 29 & 0.103 & 26 & 0.017 & 16 & 0.232 & 27 & 0.23 & 29 & 0.0776 & 25 & 0.8296 \\
\hline $\mathrm{k} 42$ & 0.17 & 27 & 0.103 & 25 & 0.0104 & 13 & 0.236 & 28 & 0.229 & 27 & 0.0779 & 26 & 0.8263 \\
\hline $\mathrm{k} 43$ & 0.17 & 28 & 0.104 & 27 & 0.0092 & 10 & 0.237 & 29 & 0.229 & 28 & 0.0781 & 27 & 0.8273 \\
\hline k25 & 0.144 & 26 & 0.114 & 28 & 0.188 & 29 & 0.212 & 26 & 0.164 & 26 & 0.0816 & 28 & 0.9036 \\
\hline $\mathrm{k} 20$ & 0.069 & 25 & 0.166 & 29 & 0.166 & 28 & 0.178 & 25 & 0.062 & 25 & 0.164 & 29 & 0.805 \\
\hline k24 & 0.386 & 30 & 0.288 & 30 & 0.529 & 30 & 0.486 & 30 & 0.464 & 30 & 0.226 & 30 & 2.379 \\
\hline k30 & 0.615 & 31 & 0.461 & 31 & 0.864 & 32 & 0.802 & 32 & 0.763 & 31 & 0.352 & 31 & 3.857 \\
\hline $\mathrm{k} 48$ & 1.11 & 33 & 0.527 & 32 & 1.34 & 35 & 0.977 & 34 & 1.44 & 33 & 0.411 & 32 & 5.805 \\
\hline k31 & 0.661 & 32 & 0.534 & 33 & 0.908 & 33 & 0.834 & 33 & 0.805 & 32 & 0.448 & 33 & 4.19 \\
\hline $\mathrm{k} 50$ & 1.33 & 35 & 0.631 & 34 & 1.6 & 36 & 1.16 & 36 & 1.72 & 38 & 0.495 & 34 & 6.936 \\
\hline k49 & 1.59 & 38 & 0.765 & 36 & 1.87 & 37 & 1.36 & 37 & 2.04 & 39 & 0.633 & 35 & 8.258 \\
\hline k32 & 2.38 & 40 & 0.646 & 35 & 0.621 & 31 & 0.587 & 31 & 2.33 & 40 & 0.657 & 36 & 7.221 \\
\hline k9 & 1.29 & 34 & 1.36 & 38 & 1.93 & 38 & 1.61 & 38 & 1.59 & 34 & 0.754 & 37 & 8.534 \\
\hline $\mathrm{k} 28$ & 1.83 & 39 & 0.858 & 37 & 1.17 & 34 & 1.05 & 35 & 1.69 & 37 & 0.822 & 38 & 7.42 \\
\hline k4 & 1.38 & 36 & 1.51 & 39 & 2.03 & 39 & 1.7 & 39 & 1.68 & 35 & 0.987 & 39 & 9.287 \\
\hline k10 & 1.38 & 37 & 1.51 & 40 & 2.03 & 40 & 1.7 & 40 & 1.68 & 36 & 0.987 & 40 & 9.287 \\
\hline k6 & 4 & 41 & 2.11 & 41 & 2.57 & 41 & 2.12 & 41 & 3.68 & 41 & 1.61 & 41 & 16.09 \\
\hline k2 & 4.86 & 42 & 3.4 & 42 & 9.18 & 42 & 4.83 & 43 & 6.3 & 42 & 2.77 & 42 & 31.34 \\
\hline k38 & 4.86 & 43 & 3.4 & 43 & 9.18 & 43 & 4.83 & 44 & 6.3 & 43 & 2.77 & 43 & 31.34 \\
\hline $\mathrm{k} 44$ & 18.9 & 46 & 7.03 & 44 & 19.1 & 48 & 3.12 & 42 & 23.7 & 49 & 4.28 & 44 & 76.13 \\
\hline k1 & 21.3 & 48 & 8.51 & 45 & 12.6 & 46 & 19.4 & 49 & 17.9 & 46 & 7.27 & 45 & 86.98 \\
\hline k11 & 23.6 & 49 & 13.1 & 49 & 19.6 & 50 & 11 & 45 & 23.1 & 48 & 10.3 & 46 & 100.7 \\
\hline $\mathrm{k} 13$ & 17.6 & 45 & 9.95 & 47 & 19.5 & 49 & 25.1 & 51 & 17.2 & 45 & 10.8 & 47 & 100.15 \\
\hline k39 & 163 & 51 & 14.5 & 50 & 14.5 & 47 & 11.3 & 46 & 144 & 51 & 15.5 & 48 & 362.8 \\
\hline k15 & 17.4 & 44 & 9.84 & 46 & 10.3 & 44 & 15 & 48 & 16.4 & 44 & 36.6 & 49 & 105.54 \\
\hline k3 & 19.9 & 47 & 11.8 & 48 & 11.5 & 45 & 12.9 & 47 & 18.6 & 47 & 49 & 50 & 123.7 \\
\hline ML & 35.6 & 50 & 22.3 & 51 & 25.7 & 51 & 19.4 & 50 & 27.5 & 50 & 52.2 & 51 & 182.7 \\
\hline
\end{tabular}




\section{Conclusion}

Simulations were varied by sample size $(n)$. The sample sizes used ere $n=50$ and $n=200$. Generally, the larger the sample size, the smaller the MSE values. It is also important to note that the sample size affects the performance of individual $\mathrm{k}$ estimators. With $n=50$ and $p=4$, the $\mathrm{k}$ estimators with the lowest total MSE values were $\mathrm{k}_{37}, \mathrm{k}_{36}$, $\mathrm{k}_{34}, \mathrm{k}_{45}, \mathrm{k}_{46}, \mathrm{k}_{8}, \mathrm{k}_{35}, \mathrm{k}_{47}, \mathrm{k}_{40}$, and $\mathrm{k}_{21}$. With $n=50$ and $p=8$, the $\mathrm{k}$ estimators with the lowest total MSE values were $\mathrm{k}_{36}$, $\mathrm{k}_{37}, \mathrm{k}_{14}, \mathrm{k}_{16}, \mathrm{k}_{12}, \mathrm{k}_{34}, \mathrm{k}_{7}, \mathrm{k}_{8}, \mathrm{k}_{45}, \mathrm{k}_{40}, \mathrm{k}_{46}, \mathrm{k}_{21}, \mathrm{k}_{35}, \mathrm{k}_{29}$, and $\mathrm{k}_{27}$. While with $n=200$ and $p=8$, the $k$ estimators with the lowest total MSE values were $\mathrm{k}_{36}, \mathrm{k}_{37}, \mathrm{k}_{12}, \mathrm{k}_{14}, \mathrm{k}_{16}, \mathrm{k}_{34}, \mathrm{k}_{40}$, $\mathrm{k}_{45}, \mathrm{k}_{46}, \mathrm{k}_{7}, \mathrm{k}_{35}, \mathrm{k}_{8}$, and $\mathrm{k}_{47}$.

Simulations were also varied by the number of explanatory variables $(p)$, with $p=4$ and 8 . It was discovered that the total MSE increases with an increase in $p$, therefore the PRR is best used when the number of explanatory variable is small.

Estimators were judged by their total MSE value for the different sample sizes, correlation, number of explanatory variable and intercept. The estimators that produced the lowest total MSE values were selected and they are as follows: $\mathrm{k}_{34}, \mathrm{k}_{40}, \mathrm{k}_{45}, \mathrm{k}_{35}, \mathrm{k}_{36}, \mathrm{k}_{12}, \mathrm{k}_{47}, \mathrm{k}_{21}, \mathrm{k}_{37}$ and $\mathrm{k}_{8}$.

In conclusion, the MSE of the estimators performed better in an increased explanatory variables $p$ and an increased intercept value. It was also observed that $k_{36}, k_{12}, k_{37}, k_{14}$ and $k_{16}$ performed better on the average at all correlation levels, sample sizes, intercept values and explanatory variables

\section{References}

[1] Alkhamisi, M., Khalaf, S. and Shukur, G. (2006), Some Modifications for choosing Ridge Parameters, Communications in Statistics- Theory and Methods, 37, (4), 544-564.

[2] Alkahamisi, M. A and Shukur, G. (2008), A Monte Carlo Study of Recent Ridge Parameter, Communications in Statistic-Simulation and Computation, 36, (3), 535-547.

[3] Algamal ZY, Alanaz MM (2018) Proposed methods in estimating the ridge regression parameter in Poisson regression model. Electron J Appl Stat Anal 11 (2): 506-515.
[4] Asar, Y. and Gen, c, A. (2017). A new two-parameter estimator for the poisson regression model. Iranian Journal of Science and Technology, Transactions A: Science.

[5] Asar Y, Gen ç A (2018) A new two-parameter estimator for the Poisson regression model. Iran J Sci Technol Trans A Sci 42 (2): 793-803

[6] Hoerl A. E., and Kennard, R. W. (1970a), Ridge Regression: Biased Estimator for Non-orthogonal Problems, Technometrics, 12, 55-67.

[7] Hoerl A. E., and Kennard, R. W. (1970b), Ridge Regressin: An Applications for Non-orthogonal Problems, Technometriccs, 12, 69-82.

[8] Kaçıranlar S, Dawoud I (2018) On the performance of the poisson and the negative binomial ridge predictors. Commun Stat Simul Comput 47 (6): 1751-1770.

[9] Khalaf Ghadban, and Ghazi Shukur (2005), Choosing Ridge Parameter for Regression Problems. Communications in statistics-Theory and Methods, vol. 34, no. 5, 1177-1182.

[10] Kibria, B. M. G. (2003), Performance of Some New Ridge Regression Estimators, Communications in StatisticsSimulation and computation, 32, (2), 417-435.

[11] Kibria B. M. G., Kristofer Mansson and Shukur, G. (2015), A Simulation Study of some Biasing Parameter for Ridge Type Estimation of Poisson Regression, Communications in Statistics- Simulation and Computation, 44, 943-957.

[12] Kibra, B. M. G., Mansson, K., Shukur, G. (2012). Performance of some logistic ridge regression parameters. Computational Economics 40: 401-414.

[13] Mansson Kristofer and Shukur, G. (2011), A Poisson Ridge Regression Estimator, Economic Modeling, 28, 1475-1481.

[14] Muniz, G., Kibria, B. M. G. (2009), On Some Ridge Regression Estimators: An Empirical Comparison, Communications in Statistics-Simulations and Computation, $38,621-630$.

[15] Qasim M, Kibria BMG, Månsson K (2020) A new Poisson Liu regression estimator: method and application. J Appl Stat.; 47 (12): 2258-2271.

[16] Schaeffer, R. L., Rio, L. D and Wolfe, R. A. (1984), A Ridge Logistic Estimator, Communications in Statistics- Theory and Methods, 13, pp. 99-113.

[17] Zaldivar Cynthia (2018), on the performance of some Poisson Ridge Regression estimator, FIU Electronic Theses and Dissertations. 3669. 